\section{A further caveat in interpreting cancer survival}

\section{Stefano Rosso and Roberto Zanetti}

We would like to take this opportunity to make some comments regarding the Perspective of de Vries and colleagues (Explanations for worsening cancer survival. Nat. Rev. Clin. Oncol. 7, 60-63; 2010). ${ }^{1}$ The authors offered a bright overview on the common pitfalls in comparing population-based survival figures that will help researchers and readers to appropriately use and interpret results of this kind. However, in addition to the presented sources of bias, it is important to mention the inclusion (or exclusion) of data concerning subsequent primary tumors, which are diagnosed following successful treatment for an earlier cancer, in survival analyses. The prolongation of life in the general population and the improved prognosis for individuals with cancer has considerably increased the possibility of subsequent primary cancers; indeed, it has been estimated that among those patients who survive a first cancer, $>6 \%$ in Europe $^{2}$ and $>8 \%$ in the USA $^{3}$ will go on to develop additional tumors.

In clinical series, survival of patients with multiple tumors is usually assessed through clinical follow-up, and specific cause of death is defined accordingly. By contrast, in series from cancer registries, clinical information on patients is generally unavailable, and assessment of the cause of death is based only on death certificates, which are often subject to misclassification. Also, in comparisons of population-based cancer survival on an international scale, common practice has been that data from patients with a history of multiple primary tumors were only analyzed for the first tumor occurrence. In this case, the probability of correctly identifying a subsequent primary tumor as multiple strongly depends on the duration of observation (that is, the length of time the cancer registry has been operational). Inevitably, unrecognized multiple cancers are included when analyzing cases from the most recently established cancer registries; this undoubtedly leads to an underestimation of survival.

The definition of a multiple primary tumor also varies, thus leading to inconsistency in the rate at which these cancers are reported. For example, Surveillance Epidemiology and End Results (SEER) rules on defining multiple primary tumors ${ }^{4}$ differ substantially from those adopted by European registries, which generally follow the analogous rules issued by the International Agency for Research on Cancer (IARC). ${ }^{5}$ For instance, SEER rules consider tumors occurring in the second organ of a bilateral pair organ (for example the breast) to be multiple primary tumors, while these are excluded according to IARC guidelines.

Moreover, the precise time of occurrence is often difficult to ascertain, leading, for example, to the opportunistic definition of synchronous cancers when diagnosed at the same time, despite uncertainty of the actual occurrence date of the cancers. Usually, synchronous tumors are excluded from survival analyses, in the belief that they represent prevalent silent tumors that have become evident during diagnostic procedures. Since the identification of another tumor can be considered similar to the presence of an important, though not-recognized, co-morbidity, inclusion of such 'nonrecognition' cases would lead to an underestimation of survival.

Brenner and Hakulinen ${ }^{6}$ reconsidered these exclusion policies, concluding that patients with a prior cancer diagnosis should generally not be excluded from comparative analyses of cancer survival between cancer registries or over time. In another study, we analyzed the amount of bias introduced, and found that inclusion of data on multiple tumors in survival estimates led to lower 5-year survival rates associated with 44 out of 45 cancers analyzed, with the greatest differences found for laryngeal $(-1.9 \%)$, oropharyngeal $(-1.5 \%)$, and penile $(-1.3 \%)$ cancers. $^{2}$

The effect of multiple tumors on survival estimates of patients with cancer is an important research field, not only because the occurrence of tumors is increasing, but also because their etiology is related to shared risk factors, including environment, lifestyle and inherited genes predisposing individuals to higher susceptibility, as well as to the delayed effects of treatments.

Piedmont Cancer Registry, Center for Epidemiology and Prevention in Oncology in Piedmont, Via San Francesco da Paola 31, 10123 Turin, Italy (S. Rosso, R. Zanetti).

Correspondence to: Stefano Rosso stefano.rosso@cpo.it

doi:10.1038/nrclinonc.2009.184-c1

\section{Competing interests}

The authors declare no competing interests.

1. de Vries, E. et al. Explanations for worsening cancer survival. Nat. Rev. Clin. Oncol. 7, 60-63 (2010).

2. Rosso, S. et al. Multiple tumours in survival estimates. Eur. J. Cancer 45, 1080-1094 (2009).

3. Curtis, R. E. et al. (eds) New malignancies among cancer survivors: SEER cancer registries, 1973-2000. National Cancer Institute NIH publication number 05-5302 (National Cancer Institute, Bethesda, MD, 2006).

4. Johnson, C. H. \& Adamo, M. SEER program coding and staging manual 2007 with 2008 revisions. National Cancer Institute NIH publication number 07-5581 (National Cancer Institute, Bethesda, MD, 2008).

5. IARC/IACR. International rules for multiple primary cancer (ICD-O Third edition). Internal report number 2004/02 (International Agency for Research on Cancer, Lyon, 2004).

6. Brenner, H. \& Hakulinen, T. Patients with previous cancer should not be excluded in international comparative cancer survival studies. Int. J. Cancer 121, 2274-2278 (2007). 